\title{
The KATRIN neutrino mass experiment
}

\author{
V.M. Hannen* for the KATRIN collaboration \\ Westfälische Wilhelms-Universität, Münster, Germany \\ E-mail: hannen@uni-muenster.de
}

The KArlsruhe TRItium Neutrino experiment aims to determine the neutrino mass in a modelindependent way from the kinematics of tritium $\beta$-decay. It combines a Windowless Gaseous Tritium Source (WGTS) with a column density of $5 \times 10^{17}$ molecules $/ \mathrm{cm}^{2}$ and a high resolution electrostatic retarding spectrometer (MAC-E filter) to measure the spectral shape of $\beta$-decay electrons close to the endpoint energy at $18.6 \mathrm{keV}$ with an unprecedented precision. KATRIN's sensitivity to the neutrino mass will be $0.2 \mathrm{eV} / \mathrm{c}^{2}$ after 3 years of full data taking. This number corresponds to an upper limit with $90 \%$ confidence level, in case no neutrino mass signal is observed; a neutrino mass of $0.35 \mathrm{eV}$ would be discovered with a $5 \sigma$ significance. At present the experiment is being installed at the Karlsruhe Institute of Technology and many components are undergoing extensive testing.

36th International Conference on High Energy Physics,

July 4-11, 2012

Melbourne, Australia

${ }^{*}$ Speaker. 


\section{Introduction}

Experimental results from neutrino oscillation experiments have established the fact that neutrinos are massive and that the neutrino flavor eigenstates $v_{\alpha}$ can actually be expressed as a superposition of mass eigenstates $v_{\alpha}=\sum_{i} U_{\alpha i} v_{i}$ [1]. However, since neutrino oscillation experiments are only sensitive to the difference of the squared masses $\Delta m_{v}^{2}=\left|m^{2}\left(v_{i}\right)-m^{2}\left(v_{j}\right)\right|$ the absolute scale of neutrino masses cannot be determined from these measurements.

Different methods have been applied to learn about neutrino masses. The most promising ones are the deduction of the overall neutrino mass density from cosmological structure formation data, time of flight measurements of supernova neutrinos, experimental searches for neutrinoless double $\beta$-decays and finally the analysis of $\beta$-decay kinematics near the endpoint energy $E_{0}$.

Kinematic neutrino mass measurements exploit the phase space dependence of the energy spectra of weak decays to extract information about the masses of the emitted neutrinos, and therefore offer a model-independent method to probe the absolute neutrino mass scale. The investigations of nuclear $\beta$-decays have yielded upper neutrino mass limits in the eV range $[2,3,4]$ that probe part of the cosmologically interesting region of degenerate neutrino masses. A lot of effort is currently put into the construction of the KATRIN experiment [5], which will push down the limit into the sub-eV range and will allow to verify claims of a mass signal from the observation of $0 v \beta \beta$ decays in the Heidelberg-Moscow experiment [6].

\section{2. $\beta$-decay kinematics}

The energy spectrum of nuclear $\beta$-decay can be calculated starting from Fermi's golden rule and has the following form [7] (with units $\hbar=c=1$ ):

$$
\frac{d \Gamma}{d E}=\frac{G_{F}^{2} \cos ^{2} \theta_{C}}{2 \pi^{3}}|M|^{2} S(E) F(E, Z+1) p\left(E+m_{e}\right) \sum_{i} \sum_{j}\left|U_{e i}\right|^{2} P_{j} \varepsilon_{j} \sqrt{\varepsilon_{j}^{2}-m_{i}^{2}} \Theta\left(\varepsilon_{j}-m_{i}\right),
$$

where $G_{F}$ is the Fermi coupling constant, $\theta_{C}$ the Cabbibo angle and $M$ the nuclear matrix element of the transition. $S(E)$ is a shape factor that takes into account the energy dependence of the nuclear matrix element. This factor equals 1 for allowed and super-allowed transitions as in tritium $\beta$-decay. The Fermi function $F(E, Z+1)$ takes into account the final state interaction of the emitted electron with the daughter nucleus and $p\left(E+m_{e}\right)$ is the phase space factor of the outgoing electron. The product of the neutrino momentum and its energy given by $\varepsilon_{j}=E_{0}-E_{j}^{*}-E$ is the phase space of the emitted neutrino, which shapes the $\beta$-spectrum near its endpoint. The neutrino phase space factor has to be summed over all final states $E_{j}^{*}$ of the daughter molecule that are populated with probabilities $P_{j}$ and over the neutrino mass eigenstates $m_{i}$. The inclusion of the $\Theta$ function in eq. 2.1 ensures that $\varepsilon_{j}-m_{i}>0$. The observable $m_{v_{e}}^{2}$ that can be extracted from the spectral shape near the endpoint is defined by an incoherent sum over the mass eigenstates weighted by the matrix elements of the $U_{P M N S}$ mixing matrix [8] known from oscillation experiments:

$$
m_{v_{e}}^{2}=\sum_{i=1}^{3}\left|U_{e i}\right|^{2} m_{i}^{2}
$$

The problem of partial cancellation of the mass terms that can arise in measurements of $0 v \beta \beta$ decays, where the observable is a coherent sum over the mass eigenstates $m_{\beta \beta}=\left|\sum_{i} U_{e i}^{2} m\left(v_{i}\right)\right|$, 

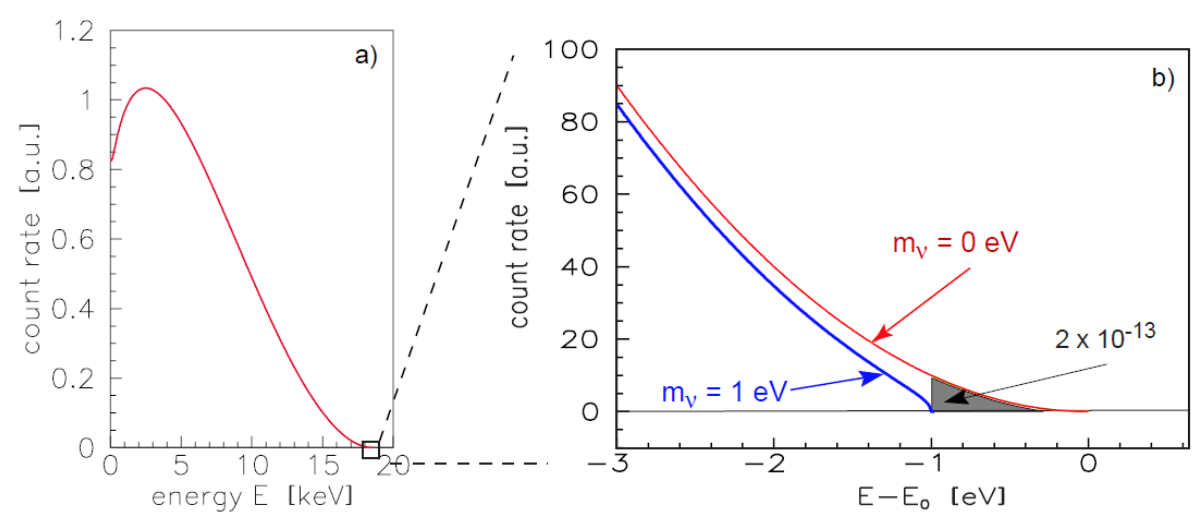

Figure 1: Energy distribution of electrons emitted in tritium $\beta$-decay (figure taken from [5]).

does therefore not occur here. The influence of the neutrino mass on the observed spectra is most pronounced in the endpoint region as shown in figure 1, where the right hand side provides a zoom into the last few $\mathrm{eV}$ of the electron energy distribution of tritium $\beta$-decay. This also highlights the biggest problem of the measurements, as in the case of tritium only $2 \cdot 10^{-13}$ of all decay electrons fall into the last $1 \mathrm{eV}$ of the distribution. The prime requirements to set up a sensitive $\beta$-decay experiment are therefore a high overall source luminosity, high energy resolution and a very low background in order to gain sufficient statistics in the endpoint region. Besides the statistics of the measurement, challenges arise in the precise treatment of systematic effects, such as inelastic scattering of electrons on their way to the detector.

\section{The KATRIN experiment}

An overview of the KATRIN experiment is shown in figure 2. The experiment works with a windowless gaseous tritium source (WGTS) where $T_{2}$ molecules are injected in the center of the

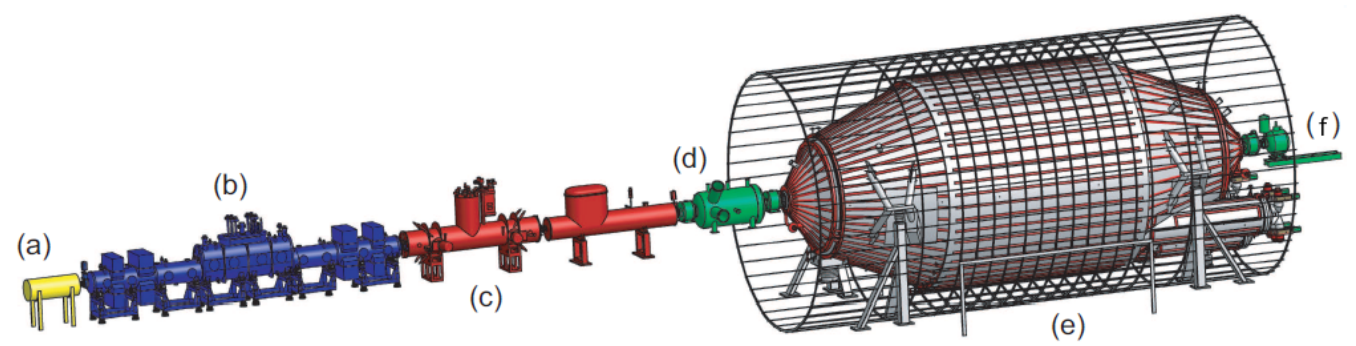

Figure 2: Overview of the KATRIN experiment. The main components are: (a) rear section calibration and monitoring system, (b) windowless gaseous tritium source, (c) transport and pumping section, (d) prespectrometer, (e) main spectrometer, (f) detector system. Overall length ca. $70 \mathrm{~m}$.

source and are removed again by turbo-molecular pumps at both ends. The $T_{2}$ gas is kept at a constant temperature of $30 \mathrm{~K}$ within the source that is operated at a column density of $5 \cdot 10^{17} \mathrm{~cm}^{-2}$. The parameters of the source are monitored by a complex sensor network and a dedicated calibration and monitoring section at the rear of the source system. About $10^{10}$ decay electrons are emitted per second in the forward direction and are guided magnetically through the transport section to the 
spectrometer tandem consisting of the pre- and the main spectrometers. The task of the transport section, made up of a differential pumping section and a cryo-pumping section, is to suppress the flow of $T_{2}$ molecules into the direction of the spectrometers by at least a factor of $10^{14}$ in order to reduce experimental background from tritium decays within the spectrometers. A first energy discrimination is performed by the pre-spectrometer which rejects the low energy part of the beta spectrum and thereby reduces the rate of electrons going into the main spectrometer to approximately $10^{3} \mathrm{~s}^{-1}$. The purpose of this reduction is to suppress background from electron interactions with residual gas molecules in the main spectrometer.

Like the pre-spectrometer the main spectrometer operates as a so-called MAC-E filter [9] and has the task to perform a precise energy analysis of the decay electrons. In a MAC-E filter electrons are guided magnetically against an electrostatic retardation potential that can only be surpassed by electrons with a sufficiently high velocity parallel to the electric field lines. The spectrometer therefore acts as a high pass filter. To reduce the amount of transversal energy of the electrons that is not analyzed by the spectrometer, the technique of magnetic adiabatic collimation is used. The idea is that the magnetic guiding field drops by several orders of magnitude from the entrance of the spectrometer to the analyzing plane, where the electric potential reaches its maximum. If the gradient of the magnetic field is small enough, such that the field is approximately constant along one cyclotron loop of the electron movement, the magnetic moment of the cyclotron motion $\mu=E_{\perp} / B$ (non-relativistic) is constant and as $B$ drops the transversal energy of the electrons is converted into longitudinal energy $E_{\|}$that can be analyzed by the spectrometer. The energy resolution of a MAC-E filter is therefore determined by the ratio of the maximum magnetic field encountered along the flight path to the minimal field in the analyzing plane of the spectrometer. In the case of KATRIN this ratio is $B_{\max } / B_{\min }=20000$ which corresponds to an energy resolution of $0.93 \mathrm{eV}$ at the endpoint of the tritium $\beta$-spectrum. By varying the electric potential of the spectrometer it is then possible to scan the relevant region around the endpoint energy of the decay and accumulate a spectrum. Electrons with sufficient energy to pass the spectrometer are detected by a 148 pixel silicon PIN detector at the end of the setup.

Among the main systematic uncertainties of the experiment are inelastic scatterings of electrons in the source, fluctuations of the source density, fluctuations of the spectrometer analyzing potential, uncertainties in the transmission function and uncertainties in the final state distribution of the daughter molecules. A sophisticated calibration and monitoring system, employing two of the world's most precise high voltage dividers [10] and a dedicated monitor spectrometer to measure mono-energetic conversion electrons from a ${ }^{83 m} \mathrm{Kr}$ source [11], is being set up to keep the afore mentioned systematic effects under control.

Another major topic that has to be dealt with in order to reach the desired sensitivity is the suppression of experimental background. To reduce the rate of background electrons created in the materials of the large main spectrometer by radioactive decays or cosmic muons, a modular, twolayer wire electrode system has been installed on the inside of the spectrometer vessel. This electrode system is kept on a slightly more negative (about $200 \mathrm{~V}$ ) electric potential than the vessel itself, such that low energy secondary electrons created at the vessel surface are reflected back into the stainless steel. Combined with the magnetic shielding of background electrons intrinsic to the operation of a MAC-E filter this will allow the background count rate to reduce to an acceptable level of $<10 \mathrm{mHz}$ [12]. Besides background suppression, the wire electrode system will be used 
to shape the electric fields inside the main spectrometer in order to achieve the highest possible homogeneity of the retardation potential, and to suppress unwanted Penning traps in the spectrometer.

The KATRIN experiment is currently in an advanced stage of construction by an international collaboration at the Karlsruhe Institute of Technology (KIT) in Germany, with many major components already on site and undergoing extensive testing. Among the recent achievements are tests of a subset of the WGTS in which a temperature stability of $<9 \mathrm{mK}$ over $24 \mathrm{~h}$ has been demonstrated for the two-phase neon cooling system of the source (the required stability is $\Delta T<30 \mathrm{mK}$ ) [13]. Other important milestones in the source section have been the commissioning of the tritium inner loop system and the Laser Raman spectroscopy system [14] to monitor tritium purity. The prespectrometer has been used as a testing ground for spectrometer related issues. After the residual background in the spectrometer has been identified as originating from ${ }^{219} \mathrm{Rn}$ decays [15] and removed by a LN2 cooled baffle, the pre-spectrometer has been moved to the main spectrometer hall. Meanwhile the installation of the inner wire electrode system of the main spectrometer has been completed and the main spectrometer is currently awaiting a complete bake-out in order to reach the required ultra-high vacuum of $10^{-11}$ mbar in the spectrometer vessel. The focal plane detector system, developed by collaborating groups from the US, has been set up and is being tested at KIT. For systematic studies of the main spectrometer properties a new kind of angular-selective pulsed UV photoelectron source has been developed [16] and will be used in the commissioning of the spectrometer.

\section{Summary}

The study of tritium $\beta$-decay close to the endpoint energy is currently the most sensitive method for a model-independent determination of $m_{v_{e}}$. While previous experiments in Mainz and Troitsk have reached upper limits of about $2 \mathrm{eV} / \mathrm{c}^{2}$ [2, 3], KATRIN aims at a sensitivity of $0.2 \mathrm{eV} / \mathrm{c}^{2}$ (95\% C.L.) after 5 years of data taking (corresponding to 3 live years worth of data) [5]. Construction and tests of the setup are under way at KIT with numerous activities taking place around the source and transport section, the monitoring systems and the recently completed main spectrometer. Commissioning of the main spectrometer is expected to take place in spring 2013.

\section{Acknowledgments}

The work of the author for the KATRIN experiment is supported by BMBF under contract number 05A11PM2.

\section{References}

[1] M.C. Gonzalez-Garcia and Y. Nir, Neutrino masses and mixing: evidence and implications, Rev. Mod. Phys. 75 (2003) 345-402

[2] C. Kraus et al., Final results from phase II of the Mainz neutrino mass search in tritium $\beta$ decay, Eur. Phys. Jour. C 40 (2005) 447-468

[3] V.M Lobashev et al., Direct search for mass of neutrino and anomaly in the tritium beta-spectrum, Phys. Lett. B 460 (1999) 227-235 
[4] M. Sisti et al., New limits from the Milano neutrino mass experiment with thermal microcalorimeters, Nucl. Inst. and Meth. A 520 (2004) 125-131

[5] J. Angrik et al., (KATRIN Collaboration), KATRIN Design Report 2004, Wissenschaftliche Berichte, FZ Karlsruhe 7090

[6] H.V. Klapdor-Kleingrothaus et al., Search for neutrinoless double beta decay with enriched ${ }^{76}$ Ge in Gran Sasso 1990 - 2003, Phys. Lett. B 586 (2004) 198-212

[7] E.W. Otten and C. Weinheimer, Neutrino mass limit from tritium $\beta$ decay Rep. Prog. Phys. 71 (2008) 086201

[8] K. Nakamura et al. (Particle Data Group), Review of Particle Physics, J. Phys. G 37 (2010) 075021

[9] A. Picard et al., A solenoid retarding spectrometer with high resolution and transmission for keV electrons, Nucl. Inst. and Meth. B 63 (1992) 345-358

[10] T. Thümmler et al., Precision high voltage divider for the KATRIN experiment, New J. Phys. 11 (2009) 103007

[11] A. Picard et al., Precision measurement of the conversion electron spectrum of ${ }^{83 m} \mathrm{Kr}$ with a solenoid retarding spectrometer, Zeitschrift fãijr Physik A 342 (1992) 71

[12] M. Prall et al., "The wire electrode system for the KATRIN main spectrometer", to be submitted to the Jounal of Instrumentation

[13] S. Grohmann et al., Precise temperature measurement at $30 \mathrm{~K}$ in the KATRIN source cryostat, Cryogenics 51, 8 (2011) 438-445

[14] M. Sturm et al., Monitoring of all hydrogen isotopologues at tritium laboratory Karlsruhe using Raman spectroscopy, Laser Phys. 20 (2010) 493-507

[15] F.M. Fränkle et al., Radon induced background processes in the KATRIN pre-spectrometer, Astroparticle Phys. 35 (2011) 128-134

[16] K. Valerius et al., Prototype of an angular-selective photoelectron calibration source for the KATRIN experiment, J. of Instr. 6 (2011) P01002 